\title{
(I) on
}

\section{THE TREATMENT OF SOME ACUTE VISCERAL INFLAMMATIONS.}

\author{
Delivered before the Harveian Society of London, November \\ 5th, 12th, and 19th, 1903.
}

BY D. B. LEES, M.A, M.D., F.R.C.P.,

Senior Physician, Hospital for Sick Children, Great Ormond Street; Physician, St. Mary's Hospital.

LECTURE II.

PNEUMONIA.

The disease which we call "pneumonia" consists in an invasion of the lungs and air passages by the micrococcus lanceolatus or pneumococcus, its rapid multiplication in this warm culture chamber, together with the efforts, more or less successful, which the human organism puts forth to resist the advance of the invading foe and to destroy it. The invader often dies in wait in the mouth of its unsuspecting host, for it can be isolated from the saliva of many healthy persons, as was first proved by Sternberg. Life to such persons is, as it were, a feast of Damocles, with a sword hanging by a hair over their heads. Too often the sword falls and the feast of life is over. A chill depresses the vital power of resistance, and the microbe runs riot in the pulmonary alveoli. Or the way is prepared by other infections, notably by the influenza bacillus, and as the influenzal infection subsides the pneumococcal infection develops, and is then of unusual virulence.

The eonflict between the invaders and the tissues may begin at one definite spot, as is usual in adults, or it may begin at the same time in several places, as' is more frequent in early life. It tends to spread rapidly over a large area of pulmonary territory, and soon becomes a pitched battle, in which all the powers of the defenders are sorely taxed to provide an adequate resistance. The blood supplies necessary for the very life of the defenders are interrupted by the stagnation in the lungs. The fight grows so intense that sleep becomes impossible, for all the vital energies are engaged. The invaders do not scruple to use poisoned weapons, and their poisons, and even some of the enemy themselves, make their way into the blood and poison the brain.

When the invaders are comparatively few or feeble, and when the resistance is powerful, the battle, after lasting from three to seven days or even longer, ends in favour of the defence, often with dramatic suddenness. The precise explanation of this remarkable phenomenon is still unknown, but the fact is familiar. For some reason or other the attack has failed, and all that remains for the exhausted defenders is to dispose of their dead foes, to clear away the débris of the battle, to cleanse the battle ground, and to restore the status quo. We have been taught to expect this happy issue of the struggle in children of more than 2 years of age, and in young adults with healthy hearts, normal livers, and sound kidneys, and it is certainly in such patients that the outlook is most hopeful. But the advent of the influenza bacillus has considerably reduced the confidence which formerly we felt with regard to the issue of a pneumonic attack in the earlier half of life, and most of us have sorrowful reminiscences of friends, strong and healthy, cut off by influenza-pneumonia in the very prime of early manhood.

Often, alas! the invaders are too powerful for the resistance, and the patient succumbs. In the first and second year of life, and again after 35 , the death-rate from pneumonia is very considerable. Dr. Hector Mackenzie says ${ }^{1}$ that pneumonia "causes very nearly as many deaths as enteric fever, diphtheria, small-pox, measles, and scarlet fever put together," and he quotes from the reports of the RegistrarGeneral the following figures showing the annual rate of mortality from pneumonia per million living at different ages:

All ages

From birth to 5 years

, 5 to ro years

, ro to 15

$\ddot{, "} 20$ to 25

", 25 to 35

", 35 to 45

", 45 to 55

, 65 to 65

Öyer 75

\begin{tabular}{|c|c|c|c|}
\hline$\cdots$ & $\cdots$ & $\cdots$ & $x, 066$ \\
\hline$\ldots$ & $\cdots$ & $\ldots$ & 3,668 \\
\hline$\ldots$ & $\cdots$ & $\cdots$ & 299 \\
\hline & $\ldots$ & $\cdots$ & II9 \\
\hline .. & $\ldots$ & $\ldots$ & 201 \\
\hline$\cdots$ & $\cdots$ & $\cdots$ & 301 \\
\hline & $\cdots$ & ... & 494 \\
\hline •. & $\ldots$ & $\cdots$ & 833 \\
\hline o. & $\cdots$ & $\cdots$ & $\mathrm{I}, \mathrm{r} 57$ \\
\hline & $\cdots$ & ... & 3,762 \\
\hline & ... & $\ldots$ & 2,596 \\
\hline & ... & $\ldots$ & 3,187 \\
\hline
\end{tabular}

He calculates that in a population of 30 millions more than 220,000 persons in each year are affected with pneumonia, and nearly 32,000 annually die from this disease. When the vitality is feeble, when the heart; liver, or kidneys are damaged by previous disease, when there are. old pleural adhesions, when the lungs are fibroid or emphysematous, when the patient is chronically poisoned with alcohol or tobacco, and when septic influences are at work, there is but a : poor chance of recovery. In many morbid states pneumonia ends the scene. Thus it becomes almost.impossible to construct statistics which could give us accurate information as to the ordinary mortality of pneumonia, or as to the results, good or bad, of any proposed method of treatment. But the outstanding fact is this-that every pneumonia is a fight for life. If we realize this, we shall never neglect its earliest stages; we shall not fold our arms in careless or unobservant "expectation," letting slip our opportunities of helping the patient in his struggle until we are confronted with a disaster which we have done nothing to avert. Can we not do something to prevent the loss-how frequent to day!-of the most important and valued lives, of fathers and mothers of families, of men and women in responsible and influential positions, whose continued life and activity may be of tremendous importance to their relatives and friends, to the conmunity in which they live, perhaps even to the whole nation?

If we are to succeed in this, it must be by the most unremitting attention, the most minute and careful observation, and by the exercise of a sound judgement founded upon previous experience. There is no disease which makes more demands upon the practitioner than pneumonia, there is none in which his action or his inaction, his wise interference or his inertia, may more affect the issue. Some cases will no doubt defy his best efforts, some would struggle through without his aid, but in a large number he can afford very great relief, and in many he may have the intense satisfaction of having certainly saved a life. But to accomplish this he must be willing to spare neither time nor trouble. He must visit his patient at least twice daily, at the most critical times he must, if possible, see him thrice in the twenty-four hours. The struggle is short and sharp. Every fresh move of the enemy must be at once detected and counteracted. The entire position of the attack and the defence must be estimated at every visit, and coming dangers must be anticipated and provided for.

In the treatment of pneumonia one thing is, unfortunately, still lacking to us. We have no specific medication. No drug is yet known to us which has an action on the pneumococcus such as that which the salicylates manifest in rheumatic infections, or that which quinine exhibits in overcoming the malarial haematozoa. Yet our reminiscence of the days when rheumatism had to be treated without salicylates should encourage us in the hope that some day we may possess a specific remedy. Neither from serumtherapy is there any present prospect of assistance. Yet here also it would be rash to predict what the future may have in store for us.

But we have to consider the position as it exists to-day. If we cannot overwhelm the foe by ordinary medication or by the subtle influence of an antitoxin, we may at least help the patient to fight his battle, and in more ways than one intervene actively for his relief. The problem is essentially how to keep him alive until the battle is over. A prolongation of life for forty-eight hours will often turn a threatening defeat into a glorious victory.

1. The Right Heart.

How, then, does pneumonia tend to kill ? Usually by cardiac failure. But this is a failure, not of the left side of the heart, but of the right; not by syncope, but by asphyxia; not through enfeeblement of the left ventricle, but through paralysing overdistension of the right.

It is remarkable how little dilatation of the left ventricle can be detected in pneumonia. Of course the pulmonary stagnation tends to diminish the tension in the left ventricle; but if the pneumococcal toxin were as injurious to the ventricle as the rheumatic, an increase of the cardiac dullness to the leit would be unmistakable. In influenza such a toxic dilatation may be often observed, and it sometimes leads to fatal syncope. This action on the left ventricle by the influenzal toxin is probably the main cause of the greater fatality of pneumonia when following an attack of influenza, as well as of influenzal broncho-pneumonia itself. In influenza the cardiac dullness may extend one and a-half or two fingerbreadths to the left of the nipple line; in a pneumococcal

[2239] 
pneumonia it does not often extend much beyond the nipple ine and even this moderate increase seems often to be caused by the distension of the right side of the heart, for it may subside at once after a bleeding. Thus it seems that the pneumococcus and its toxin do not attack the ventricular wall so actively as the influenza bacillus or the rheumatic diplococcus.

It must of course be remembered that the left ventricle may be found enlarged as the result of previous disease, rheumatic or otherwise, as the effect of strain or of arteriosclerosis or granular kidney. In any case it adds gravity to the prognosis.

But the right side of the heart is in pneumonia very soon overburdened by the pulmonary stagnation; and it quickly shows signs of distress. Within forty-eight hours from the onset evidence of dilatation of the right auricle can often be detected by percussion in the fourth right space. Unless means be adopted to relieve it, the right side becomes increasingly embarrassed as the days of the terrible pneumonia week pass by. The normal fingerbreadth of dullness in the fourth right space becomes doubled, perhaps more than doubled, and duliness may be found in the third space as well. The first symptom caused by the distension of the auricle is an increase in the rate of the respiration. At a later stage this is accompanied by lividity of the lips and cheeks, with pallor, increasing to a general cyanosis, due largely to the interference with oxygenation in the lungs. As the tension in the right heart increases, the patient feels much oppression and distress, and often becomes very restless, seeking ease in change of position and not finding it. This rightheart misery may be quickly relieved by a little loss of blood. In an early stage from three to six leeches will at once remove the distress, and the dullness of the right auricle rapidly diminishes to the normal fingerbreadth. The relief will usually last for forty-eight hours, after which time renewed distension of the right side may again demand relief. In a severe case it may be advisable to use leeches three times during the week, but in many cases two applications of leeches will suffice to keep the right auricle from paralysing overdistension until the crisis.

An old lady of 73, the mother of a Fellow of the Royal College of Surgeons, was seized with pneumonia of moderate severity. At first all went fairly well, but on the fifth day her pulse began to show intermissions, the lips became blue, the respiration-rate rose to 40 , and she became very restless. The next morning (sixth day) she told me that she was very ill and was going to die. I found that the right auricle extended two fingerbreadths in the fourth right space. Her son at once saw the necessity for some removal of blood, in spite of her advanced age, and eight leeches were applied over the lower right ribs. In the evening she was again quite cheerful, and I found that the dullness of the right auricle had nearly returned to the normal fingerbreadth. Three days later she again had a very restless night and said that she was going to die. Again I found the right auricle dilated. Three leeches only were enough to give relief on this occasion. Next day she was out of danger, and recovery followed. Some weeks later I chanced to meet her in the street, when she volunteered the remark, " You know, doctor, those blackies did me good!"

By way of contrast let me mention the case of a much younger lady whom $I$ saw in consultation and found to be suffering from pneumonia. No attention had been paid to the right heart; it was much dilated, and the condition of the patient critical. Feeling sure that it would be useless to propose a venesection, I requested the practitioner in charge to apply leeches. His reply was that he would "see if any could be obtained in the neighbourhood." The patient died.

If the early leeching be omitted the right auricle may become, as in this case, much distended, and right-heart misery may be marked. In this condition it is better to have recourse to venesection and to remove $6 \mathrm{oz}$. or $8 \mathrm{oz}$. of blood. A larger quantity should be taken when the dilatation of the right heart is great, the pulmonary consolidation extensive, and moist râles heard over the rest of the lungs. In such circumstances $18 \mathrm{oz}$ : or $20 \mathrm{oz}$ of blood must be taken if the patient is to have a chance of life. I have seen life saved even at this stage by a venesection to $18 \mathrm{oz}$. The blood obtained by venesection is often very dark in colour, and it sometimes spurts out as if from an artery when a vein is opened, showing the increased intravenous tension, and proving the need for relief of the intra-auricular tension.

In using the amount of dullness in the fourth right space as a guide when considering whether or not to bleed it is necessary to be careful not to overestimate the size: of the auricle, owing to pulmonary consolidation in its immediate neighbourhood or to pleural effusion, and, on the other hand, not to underestimate it through a local hyper-resonance of lung caused by compensatory hyperdistension of parts of lung not yet implicated in the inflammatory process. Of the two errors, probably the latter is the more serious, for it may tend to postpone a needed relief to the heart. In case of doubt the practitioner will be wise to decide for the leeches; they can hardly do any harm and they may do much good.

The total amount of blood removed by even threeapplications of leeches or by a moderate venesection is really quite small. The plan here recommended is something very different from the excessive bleedings of former days and the purpose is different. It is not with the idea of controlling an inflammatory process, but with the intention of giving relief to an overstrained right heart.

The repeated bleeding of cases of pneumonia advocated and practised sixty years ago was based on theoretical considerations. But it would hardly have been continued so persistently if some benefit had not been observed to follow its first employment in most cases. Doubtless this benefit was the relief of the right heart unintentionally produced and the consequent diminution of dyspnoea and restlessness.

The most important part, then, of the treatrnent of pneumonia is to keep a constant watch on the condition of the right heart, and to prevent its overdistension by the timely removal of a small quantity of blood. This indication is, unfortunately, too generally neglected. The omission is probably responsible for a large part of the mortality from pneumonia. If a patient suffering from distension of the urinary bladder came under the care of a medical man he would be at once relieved. But if it be the patient's right heart that is distended, a condition causing equal misery, equally dangerous, equally capable of being diagnosed, and equally easy of relief, the patient will probably be left to take his chance, because the practitioner's percussion is inadequate and bleeding is out of fashion. No improvement in the physical examination of patients is more needed than an improved percussion of the heart; no reform in therapeutics is more urgent than a general adoption of the practice of moderate blood letting in some diseases of the heart and of the lungs.

It is obvious that the diet during an attack of pneumonia must be such as can be taken without effort, such as can be easily digested, and such as shall readily be assimilated. Milk fulfils all these indications ; it has also somewhat of a diuretic influence, and thereby will assist the elimination of toxin.

But there is one point which needs consideration. The introduction of a fluid nutriment in large quantities will tend to increase the quantity of fluid passing through the right heart, and this is a matter of importance when that organ is already overstrained. For the first two or three days of the disease milk alone, in quantities of three or four pints daily for an adult, should be given. But when the right auricle is becming dilated it is wise to administer small quantities of a highly concentrated and predigested nutriment. A useful resource at this time may be found in malted milk, a dry powder one half of which consists of desiccated milk, the ther half of malted wheat and malted barley, with a little sodium and potassium bicarbonate: Half an ounce of this dissolved in $2 \mathrm{oz}$. of milk may be given every hour while the patient is awake; for a child the amount may be two teaspoonfuls in $\mathrm{roz}$. of milk. Thirst will probably not be feIt for a day or two, and it may then be satisfied in another way, for as soon as the first leeches have relieved the right heart it is possible to administer without harm considerable quantities of water. As much as 4 pints of water may be given in divided doses of half a pint every three hours during the day following the relief by leeches or venesection. This not only satisfies thirst but it helps to wash out the pneumococcal toxin, and thus is of great value. I have seen this measure produce a most marked effect in removing the delirium in a severe case of typhoid, and it has a similar influence in pneumonia.

\section{Sleep.}

In pneumonia sleep may be disturbed from the first by pain in the side; afterwards by continued pain, by dyspnoea and restlessness, by fever and rapid pulse, finally by cerebral congestion; and even by pneumococcal invasion of the meninges. Yet the refreshment, of sleep is most important if the patient is to fight a winning battle; and the lack of it sadly diminishes hís energy and power of resistance. Every 
night's sleep is of importance. Restless and disturbed nights in the early part of the attack are apt to be too little regarded by the practitioner in attendance. When the fourth or fifth comes, and the need for sleep is urgent and distressing, he dares not administer hypnotics because of the danger of inducing a fatal coma. Whenever a medical man is called in at the onset of an attack of pneumonia, let him never forget to make sure that the patient sleeps during the first three nights of his illness. Whatever other treatment he may adopt, or refrain from adopting, let him take measures to diminish pain and to secure sleep. If there is pain which cannot be otherwise relieved, morphine by injection under the skin is necessary, and at this early stage is perfectly safe. If pain is slight or has been relieved, a dose of bromide and chloralamide, or of trional, given in hot brandy and water, will suffice. But pain must be relieved and sleep secured.

When the right heart is overstrained the procuring of sleep is a difficult and anxious question. When dyspnoea becomes distressing it is almost impossible for the patient to sleep; all his energies are needed for the maintenance of the respiratory function. Morphine given at this time diminishes the activity of the respiratory centre, on the energy of which his life de pends, and may induce a coma which will end in death. The way out of this dilemma is found in a relief of the right heart.

After leeches or venesection the urgency of the dyspnoea subsides, the cyanosis diminishes, comfort is restored, and the wearied patient may fall asleep without any narcotics. This effect is often most marked and most encouraging. But, if necessary, a small dose even of morphine may now be given with safety, or other hypnotics if pain be absent. The double effect of the relief to the heart and the rest to the nervous system gives the patient an enormous advantage in the struggle which still lies before him. Even at a late stage of a severe case morphine may sometimes be safely given, provided that the right heart has been relieved and the air passages cleared by a venesection an hour or two previously. But where a careful watch has been kept on the right hèrt, and it has been duly relieved by leeches, and at the same time a vigorous attack has been made on the inflamed areas in the lungs by the free use of ice externally, morphine is hardly ever necessary, unless the pleura is severely involved and an empyema is forming.

\section{Cardiac Tonics.}

Medicines which assist the heart to maintain the circulation are often of much service in the later days of a pneumonia. It is generally advisable to begin the use of these about the third or fourth day of the disease, and they ought to be given, if at all, somewhat freely.

Of these drugs, strychnine is probably the most useful, It should be given by subcutaneous injection. Half to one minim of the official solution twice daily should be the initial dose for a child; for an adult 2 minims morning and evening at first, increasing to 5 minims if necessary. Even larger or more frequent doses may be given with advantage in influenza with dilatation of the left ventricle.

Atropine by subcutaneous injection is also very serviceable in children, but not so useful for adults because they suffer much more than children from the dryness of throat and other unpleasant effects of belladonna. In children large doses of this drug will cause chiefly flushing of skin, which is of no importance. If the dose be further increased there may be dilatation of pupil, some mental exoitement and restlessness, in some cases diarrhoea. The subcutaneous in jection of I minim of the liquor atropinae twice daily, increased to every four hours, is often of the greatest possible service in the cardiac failure of diphtherial paralysis. I have used it for many years and am convinced that it has often saved life. But it must be given freely and one must not be afraid of a little delirium. Small doses are useless in a condition like this. Strychnine may be given with advantage at the same time, but I am sure that the atropine is the more useful. In the paroxysmal dyspnoea of mitral stenosis, with rapidlyincreasing breathlessness, pallor, and sometimes evidence of dilatation of the right auricle a subcutaneous injection of 4 minims of liquor atropinae will often cut short the attack in a few minutes, and in children and adolescents will cause, as a rule, tho unpleasant consequences. The same treatment is sometimes effectual in older patients, but in them the afterresults of belladonna are much more troublesome.

In the pneumonia of ehildren belladonna has been strongly recommended by Dr. Coutts, and Dr. Eustace Smith has told me of an apparently hopeless case of pneumonis in a child in which recovery followed the free use of belladonna, the child being delirious for three days. The tendency of the drug to cause delirium is a great disadvantage in using it for pneumonia, which itself is very prone to cause delirium. On the whole it is a remedy much more useful for children than for adults.

Oxygen by inhalation assists the aerration of blood in the lungs, and thus improves the quality of the blood supplied to the cardiac muscle. It is therefore truly a cardiac tonic. Its use should be begun as soon as cyanosis is definite, and should be continued for five minutes every hour, whether the patient is awake or asleep. It can be given without disturbing him in the least. Uxygen is certainly a most valuble remedy, and ranks with strychnine in the treatment of pneumonia. But neither strychnine nor oxygen, nor both together, will often save life if the right auricle be not relieved. After a bleeding they are powerful remedies; without removal of blood they often fail, and almost necessarily. It is good to maintain the strength of the cardiac muscle; it is still better to diminish its labour. It is best to do both.

Digitalis will not always reduce the frequency of the pulse in pneumonia, especially when the temperature is high. It is most likely to be of service after relief of the right heart, when the fever is moderate and the pulse still remains weak and frequent.

Ammonium carbonate may be given when there is evidence of much secretion in the bronchial tubes.

Alcohol, though called a "stimulant," has not much title to be considered a cardiac tonic. It is essentially a vasomotor depressant, and as such may help the heart indireetly when the tension is high. There is also sometimes a temporary increase in the strength of the puls 3 after the administration of a moderate dose, probably due to increased blood supply to the cardiac muscle, through relaxation of eoronary arterioles. It is therefore possible that repeated small doses may be of service in pnoumonia, but the large doses sometimes advised ane likely to do more harm than good. To imagine that brandy can "support" the heart when the right side is becoming paralysed from overdistension is absurd. In such a case the only satisfactory cardiac tonic is a venesection.

$$
\text { 5. Ice. }
$$

In pneumonia the application of ice to the wall of the chest is as helpful as it is in pericarditis, but it must be used much more freely. A single icebag will cover the whole anterior surface of the heart, but the lung is a much larger organ, and for the efficient treatment of an inflamed lung at least two icebags, often three, are required. If both lungs are attacked four icebags may be necessary in the case of an adult. It is, therefore, all the more important to keep in mind the two precautions which I have already mentioned: the feet and lower limbs must be kept continuously warm by means of hot-water bottles and the right heart must not be permitted to be overdistended. The temperature should be taken and recorded every two hours; in the case of a young child this should be done every hour. Of course the most efficient nursing is required and should be provided from the first.

The parts of the lung which are inflamed must be determined by careful percussion and outlined on the chest with a blue pencil. The icebags must be carefully kept in contact with the skin over the areas thus determined by passing their screwtops through holes in a binder loosely fastened round the chest, or a vest may be cut out of domett as above advised in pericarditis. Special care must be taken that there is no leaking, also that they are surrounded by cotton-wool or a soft towel, and a pretty thick padding of such material is needed when the patient has to lie on the icebags applied to his back. This is really the greatest difficulty in the use of icebags in pneumonia; the patient hardly ever dislikes the cold, but the pressure of the small lumps of ice on the back as the patient lies upon the bags sometimes taxes the skill of the nurse to arrange matters comfortably.

At least once daily, preferably twice, the practitioner must percuss the whole of both lungs as well as the heart. This must be done systematically and with care. Begin with the heart and determine the limit of its dullness to the left, and especially its limit to the right in the fourth and third intercostal spaces. Notice the strength of the impulse of the left ventricle and the force of the epigastric pulsation of the right ventricle. Listen to the quality of the first sound and observe the strength, rate, size, rhythm, and length of the pulse wave. Notice whether the aortic second sound, and especially the pulmonary second sound, is too loud or too feeble. Then percuss carefully the front of the lung and the upper axilla of 
each side from above downwards quite down to the anterior base, carefully noting and marking the limits of any areas of dullness. . Listen for crepitant sounds, especially inspiratory crepitation, and for bronchial or tubular breathing, but do not fatigue the patient by attempting to elicit bronchophony. All this can be done in a few minutes. Then turn the patient over on his right side and carefully examine the back of the left lung from above downwards and the axillary region, marking out all dull areas as before. Let the patient rest for a minute or two on his back, and then turn him over on his left side and examine in a similar manner his right lung and right axilla. You have now a fairly accurate idea of the extent and distribution of the areas of inflammation. In children these are almost always multiple and scattered over both lungs, some of the dull areas being partly due to collapse. But even in children the inflammation may tend to spread chiefly at one spot, and to involve a large part or even the whole of one lnng, while the other is comparatively little affected. Yet, even in such so-called "lobar" cases careful percussion almost always yields areas of dullness in the opposite lung. And this is of great importance, for if such areas are allowed to spread, they may develop into such extensive areas of inflammation that the most careless percussor cannot fail to notice that he now has to deal with a "double pneumonia."

In adults the inflammation is usually more localized than in children, but there is the same tendency to spread. This extension of dullness can be easily watched by careful percussion practised daily, and a very good idea of the intensity and virulence of the inflammation may thus be obtained. This is requisite for the efficient use of the icebags. It is impossible to surround the whole of both lungs with ice; it is therefore necessary to determine where the lung is inflamed, and in which direction the inflammation is spreading, and to apply the ice over precisely those areas. Place the first icebag on the largest dull portion of lung, and apply a second bag behind or in front, so as to enclose the inflamed area. These ice applications are generally very pleasant to the patient. An old ladv of 62, after a few hours' trial of an icebag, declared it to be "delicious." A young lady of 19 said that it was "lovely," and when asked whether she wished it continued, replied with emphasis, "Oh, rather! yes!" Nor is there usually any difficulty in persuading the patients to try this method, for they have generally already tried hot applications and have found little or no relief. With a temperature of $104^{\circ}$ it is easy to understand that cooling means comfort. The difficulty is not with the patients, but in overcoming the prejudices of the patients' friends.

At the next visit of the practitioner after the application of the icebags let him remove them, and carefully percuss again the areas to which they have been applied. He will generally find that they are less dull than on the previous occasion, and that the air enters them in inspiration better than before. He may even find that the dull area is somewhat smaller. Encouraged by this observation he will replace the icebags, and will proceed to attack any other dull area that he can find by means of a third icebag. At the next visit he may find that the areas attacked show signs of further improvement, or at all events have not extended. But the inflammation may at the same time be spreading elsewhere; new spots of dullness may have appeared, or dull areas formerly small may be rapidly extending. This should be suspected especially when the temperature continues high in spite of the application of the ice, or when after falling it again rises. Then let the practitioner make diligent search for fresh and extending patches of dullness; he will often find them, perhaps in the lung he had thought unaffected. Care in this matter is of the greatest importance to the patient, for if such patches be detected in their earliest stage it is remarkable how quickly they will yield to the local influence of the ice, but if they are overlooked or neglected they often increase rapidly, and may become extremely serious. It is specially desirable to have the ice over the spreading edges of the inflamed areas as far as this ean be managed. It is often necessary to shift their position, more or less, at least twice daily, sometimes more frequently. It may be needful at every visit to give fresh directions to the nurse as to the exact areas to which the ice is to be applied. This is one reason why in pneumonia two visits daily are essential, three sometimes desirable. The progress of the conflict must: be watched over the whole battlefield, during the whole struggle; by the general in command; if he is to secure the victory. The right heart and both hungs must be most carefully examined in detail at least once daily, a second time if the strength of the patient will allow it. It may sometimes be better to omit a minute examination at the evening visit, but at the morning visit the whole ground should be carefully gone over.

Thus far I have advocated the use of the icebag in pneumonia solely on the ground of my own experience of its practical utility. But something may be said in its favour from the standpoint of bacteriological science. For bacteriologists find that the pneumococcus is remarkably susceptible to changes in its environment, and in particular to changes of temperature. Slight alterations in the composition of the culture media will affect its growth, so that it is very difficult to cultivate. Dr. Eyre, who with the late Dr. Washbourn has specially studied this organism, tells $\mathrm{us}^{2}$ that it is " extremely susceptible to variations of temperature." He adds that " the range of temperature enjoyed by the pneumococcus is limited to about $14^{\circ}$, and is bounded by $28^{\circ} \mathrm{C}$. on the one hand, below which no growth takes place, and by $42^{\circ}$ C. on the other. The optimum temperature is undoubtedly $37.5^{\circ} \mathrm{C}$." It is therefore not unlikely that persistent local reduction of temperature of a pneumonic lung, even though of moderate amount, may exercise a definite inhibitory influence on the rate of multiplication of the developing pneumococci. Fortunately this is specially likely to be true in the worst cases of the disease, for Dr. Washbourn ${ }^{3}$ showed from his experiments that the greater the virulence the pneumococcus is found to possess the more marked is its susceptibility to surrounding influences, and the more difficult it is to cultivate. Less virulent "strains" of this organism were able to grow at lower temperatures. He found that a pneumococcus which was slightly virulent, and which would grow on artificial media at a temperature as low as $20^{\circ} \mathrm{C}$., could by passages through animals be converted into a highly virulent type, which would not grow on artificial media at temperatures below $37^{\circ} \mathrm{C}$. If this is so it suggests that the ice treatment may have a special utility in the worst types of pneumonia.

It is difficult to estimate the amount of local reduction of temperature caused by the persistent application of ice over an inflamed lung, and there seem to be no available observations on the comparative rate of growth of the pneumococcus at temperatures differing from each other by $5^{\circ}$ or $10^{\circ}$. I therefore requested Mr. Neave, Bacteriologist to the Hospital for Sick Children, to make some investigations with regard to this. Mr. Neave has kindly taken much interest in this research, and his report is as follows :

"Some months ago I undertook at your request to do what I could to test the cultivation of the pneumococcus at the temperature of the blood, and compare the growth with that at slightly lower temperatures. I confess the undertaking was made with a lighter heart than the exigencies of the matter really warranted, and that the results arrived at. to say the least, are far from complete. It would appear that each strain of the organism varies enormously in its idiosyncrasies of growth, and that a change is effected after each cultivation on a medium which has been differently prepared in some minute particular. The great variety of statements as to the nature and behaviour of the pneumococcus, I feel sure, is due to these inherent differences. It is open to question what exact value can be put upon the planting of a number of organisms, and counting those that grow and make colonies. The late Dr. Washbourn, in his Croonian Lectures, has shown that in cultivations that appear defunct, if a very large quantity is planted, one or two of surpassing vitality may yet be found to grow. I have, however, not been able to think of any better method of testing the effect of temperature on the growth of this organism.

'In the first place, I found that the isolation in pure culture of this organism was only occasionally possible, and that pleural effusion strains, which were the most easily obtainable by me, had hardly ever sufficient vigour of growth on artificial media to enable a second or subculture to be made' on a solid medium. This was necessary for the purpose of inoculating a plate with a definite quantity of liquid medium containing a growth of the organism. These difficulties I find well described in the Baumgarten Jahresbericht, 1900. 'However, by the kindness of Drs. Klein and Gordon, an opportunity was given for the following:-A colony isolated from sputum was injected into mice, and the peritoneal and subcutaneous fluids resulting proved to be of exceptionally vigorous' growth. 'In the' first plating the peritoneal fluid was diluted by sterile saline solution, and an equal measured quantity was spread by a sterile glass rod on two agar plates. 
One was incubated at $39.4^{\circ} \mathrm{C}$. the other at $32^{\circ} \mathrm{C}$., resulting in 285 colonies at the higher temperature and 15 colonies at the lower. It would have been far more satisfactory to have used the same liquid at the other temperatures required at the same time, but there were only two incubators at my disposal. In consequence of this, a fresh cultivation had to be made every two days and used anew for each subsequent comparison. Thus no comparison between one pair of groups and another can be made. In the second comparison one plate was incubated at $37.5^{\circ} \mathrm{C}$. and produced $\mathrm{I}, 568$ colonies, while the second at $27.5^{\circ} \mathrm{C}$. produced 690 colonies. In the third, $38.5^{\circ} \mathrm{C}$. produced 153 colonies, and $3^{\circ} \mathrm{C}$. produced 42 colonies.

"The above, although consisting only of one set of experiments, conclusively proves in respect of that particular strain of the organism that a temperature of a few degrees less than that of the body prevents so active a growth.

"It may be that the comparatively more vigorous growth at the lower temperature in the later experiments was due to the organism having been brought up on artificial media, and so had become less virulent. It may be noted that Dr. Washbourn points out that the avirulent varieties have a stronger growth at lower temperatures than those of a virulent character."

These observations appear to indicate that a moderate reduction of temperature does exercise a marked inhibitory influence on the rate of growth of the pneumococcus. If they are confirmed by subsequent and more extended investigations they will furnish a scientific justification of the employment of the icebag in the treatment of pneumonia.

Whether or not this scientific basis can be claimed for the treatment any one who will thoroughly and carefully employ it in the manner already detailed, and with the precautions above mentioned will soon be convinced of its practical usefulness. Any estimate of its value, founded on the use of one icebag only, in cases seen only two or three times in the week, and without any careful percussion of the right heart and any attempt to relieve it, is worthless.

One striking fact which ought not to escape notice is the great improvement which an efficient ice treatment often produces in the physical signs before the crisis. This may be very distinct even two or three days before the crisis, and already very great indeed when it occurs. Another striking fact is the more rapid convalescence. This is natural enough, for if the microbic growth is inhibited there is less poisoning of the tissues. less dead material to be absorbed and eliminated, and less diminution of the patient's strength.

One point remains to be noticed. Some physicians look upon pneumonia as a blood disease with local lesions, and would, therefore, consider the icebag treatment as irrelevant and useless. Now it is true that occasionally pneumococci have been detected in the blood of pneumonic patients, but Dr. Washbourn held that it is only in a few severe cases that this can be done. This difficulty in detection indicates that the number in the blood is comparatively small. In whatever way the organisms may have reached the pulmonary alveoli in a case of pneumonia, whether by inhalation or by the blood current, it is certain that their chief, probably in most cases their only, seat of multiplication is the lungs. From the lungs they no doubt often pass into the pleura, sometimes into the pericardium, occasionally into the blood current, especially in the worst cases.

Dr. Washbourn has proved experimentally that it is possible for pneumococci introduced into the air passages to pass through the lungs and produce pleurisy and pericarditis without any implication of the lung tissue itself. Clinically such cases are met with, but they are much rarer than pneumonia. Yet it remains true that the chief site of pneumococcal growth is in the air cells of the lungs, and that the congestion and the production of fibrin are the local results of this growth and of the toxins thereby produced. On these points there is some valuable information in Dr. Auld's Selected Researches in Pathology, published in 1901. He says: "If a pure, moderately virulent culture of the pneumococcus be inoculated either subcutaneously or into the internal cavities of a susceptible animal, it produces around the site of inoculation intense congestion, exudation of sanguineous serum, and fibrin. That is its characteristic and invariable lesion, and it produces directly no other. Should resolution not occur soon, we have, of course, after a variable time, an exudation of leucocytes, the gradual collection of which may give rise ultimately to' what has the ap pearance of a mass of pus."

By inceulating beneath the skin of a rabbit rusty sputum from a case of pneumonia, and killing the animal within thirty-six hours, Dr. Auld found that its heart blood contained a pure culture of the pneumococcus. He grew these on solid and liquid media, and made injections into the pleural cavity of rabbits, causing always a severe pleurisy, irequently severe congestion of the lungs, often more or less consolidation, in two cases pneumonic consolidation of an entire lobe, usually also pericarditis. The local lesions, lungs, and spleen of these rabbits were subjected to a chemical analysis, and from them were obtained an albumose and an organic acid. The latter gave the reactions for lactic acid; it was not toxic on injection into animals. Dr. Auld suggests that the production of this acid may have an inhibitory cffect on the further growth of the pneumococcus.

But the albumose acted very differently. When injected beneath the skin of the rabhit's ear, local inflammation of the ear was produced and a rise of temperature ; recovery followed. Intravenous injection produced initial shock, followed by a marked rise in temperature, but no other pathological effect. Injection into the pleural cavity produced no initial depression, but marked dyspnoea soon set in, followed by rise of temperature. The animal was killed on the third day, and found to have right-sided pleurisy, with complete consolidation of the lower lobe of the right lung. No other lesions were found. Inoculations were made from the blood, and also from the serum in the pleural cavities in solid and liquid media, with a negative result. A larger quantity of the albumose was also intrapleurally injected in another rabbit. Next day the temperature was $104.6^{\circ}$. On the following day the animal was killed, and severe right lateral and also diaphragmatic pleurisy was found, with pericarditis and hard consolidation of the upper part of the lower lobe of the right lung, very typical of ordinary lobar hepatization.

Thus it seems that the consolidation of lung in pneumonia is largely due to the local effects of an albumose produced by the growth of the pneumococcus, and that the pyrexia is due to the same cause.

A similar albumose was obtained, in smaller quantity, by growing the pneumococcus in albuminous media free from albumoses, and it was found to produce similar results on injection into rabbits, thus proving that the pneumococcus has the power of forming a highly toxic albumose from proteid material. Much larger doses, however, were required. showing that the production of toxin is much more active in the living body than in artificial media.

Another interesting observation of Dr. Auld's may also be mentioned. He found by injection of pneumonic toxins obtained by filtration of cultures of the pneumococcus that the effect of such injections was to make the animals subjected to them more susceptible to subsequent infection with the pneumococcus than normal animals. This susceptibility appeared to last for a long period. The clinical importance of this fact is obvious. R Practilioner, 1900, p. ${ }_{36}$. 2 Ibid, s9oo, p. 285.
1900.

\section{SYPHILITIC JOINT DISEASE.}

By E. PERCY PATON, M.S., M.D., F.R.C.S., Assistant Surgeon, Westminster Hospital.

Thovar syphilitic disease of the joints is not of very frequent occurrence it is not improbable that it is somewhat more so than is generally supposed, as it is not difficult to see how it may be either overlooked or mistaken for some other affection, such as osteo-arthritis, and it is probably still true, as Professor Howard Marsh ${ }^{2}$ says in his work on the Diseases of Joints, that this trouble has hardly received the attention from English surgeons that it deserves.

A number of these cases having come under my notice, and as I have also looked somewhat closely into the literature of the subject and examined the few specimens that are to be found in the museums in London illustrating the morbid anatomy of the disease, I have thought it might be of service to put the results on record.

That syphilis-or, at any rate, venereal disease-might affect the joints seems to have been known to Petrus Martyr in 1488; the disease is also referred to by Alphonsus Ferrus in 1537; and by Morgagni and Fabre in the seventeenth century. Hunter, however, thought that syphilitic disease did not occur ; in this view he was opposed by others of his time, but the earliest clear account of the disease seems to have been given by Richet ${ }^{2}$ in 1853 . 